\title{
Blowfly Larval Tissues as a Secondary Detector for Determining Paraquat-Related Death in Rabbit Carcass*
}

\begin{abstract}
Paraquat poisoning is commonly associated with suicide or homicide in Malaysia. In a case involving advanced body decomposition, pathological analysis regarding the cause of death may become difficult or almost impossible. Insects serve as common alternative matrix for poison detection in forensic analysis. Paraquat detection via secondary bioaccumulation in fly larvae tissue has never been reported. In this study, tissues from blowfly larvae collected from a rabbit carcass with paraquat poisoning were analyzed for secondary bioaccumulation. Larvae samples were collected and analyzed using liquid-liquid extraction. The detection was performed via reduction of quaternary ammonium presence in paraquat and analyzed using gas chromatography-mass spectrometry (GC-MS) with selected ion monitoring mode (SIM mode). GC-MS showed the elution of reduced paraquat was at retention time 12.8 min. Blowfly larvae tissue has proven useful as a secondary detector in paraquat-related deaths.
\end{abstract}

KEYWORDS: forensic science, entomotoxicology, paraquat, poisoning, gas chromatography-mass spectrometer, blowflies

Paraquat dichloride (PQ) is a dipyridylium herbicide widely used in agriculture and aquatic crops as plant growth regulator and crop defoliant. It is a highly toxic chemical and causes lethality when ingested or when in contact even in a small amount. Fatal cases of accidental, suicidal, and homicidal poisonings with PQ have been reported worldwide (1).

Suicidal cases involved PQ are not rare issue in Malaysia. From 1979 to 1988,577 cases $(27.8 \%)$ related to PQ poisonings have been reported from the total of 2,076 cases of human poisoning in Malaysia (2). Deaths from pesticide poisoning make a major contribution to patterns of suicide in developing countries, particularly in rural areas. The reported substances that are most commonly used for self-poisoning are agriculture pesticides, which are detected in 10-20\% of fatalities (3).

Deaths connected with suicide by intake of poisons are sometimes not discovered for a period of time, which results in lack of conventional matrices for toxicological analysis. This complicates the forensic investigations (4). The use of insects as alternative matrices as well as a secondary detector of poisons in decomposing tissues is well established $(5,6)$.

Larvae of flies that are found consuming a body may ingest, incorporate, and bio-accumulate chemical metabolites of drugs or toxins from the corpse into their own tissues (7). Necrophagous insects are the most reliable sources for toxicological

\footnotetext{
${ }^{1}$ Department of Chemistry, Faculty of Resource Science and Technology, Universiti Malaysia Sarawak, Kuchig, Sarawak 94300, Malaysia.

${ }^{2}$ Faculty of Medicine and Health Science, Universiti Malaysia Sarawak. Kuchig, Sarawak 94300, Malaysia.

*Funding support provided by the Ministry of Energy, Water and Communications, Malaysia (Program Mentor: 1IPTA 1MENTERI).

Received 1 July 2014; and in revised form 28 Oct. 2014; accepted 7 Nov.
} 2014.

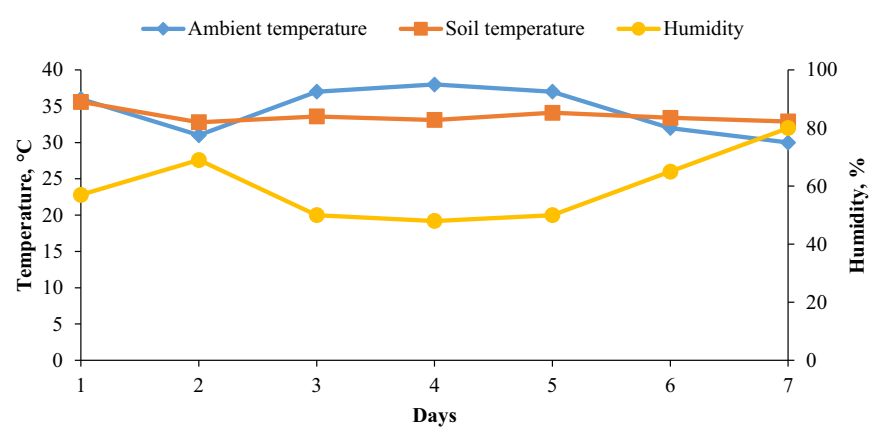

FIG. 1-Temperature data and humidity on the experiment.

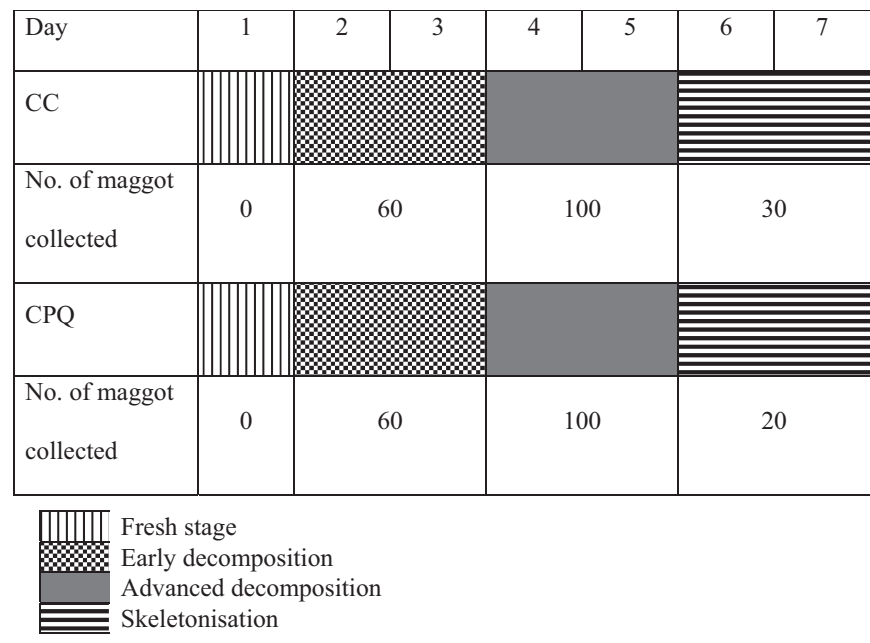

FIG. 2-Decomposition stages of control carcass (CC) and carcass ingested with paraquat dichloride $(C P Q)$ as well as the maggots collected. 